\title{
Fault Estimation for A Class of Discrete-Time Nonlinear Systems *
}

\author{
Z. Wang ${ }^{*}$ M. Rodrigues ${ }^{* *}$ D. Theilliol ${ }^{* * *}$ Y. Shen ${ }^{*}$ \\ * School of Astronautics, Harbin Institute of Technology, Harbin, \\ 150001 P. R. China (e-mail: z.h.wang@hit.edu.cn). \\ ** Automatic and Process Control Laboratory, University of Lyon, \\ Lyon, F-69003,France (e-mail: mickael.rodrigues@univ-lyon1.fr) \\ *** Centre de Recherche en Automatique de Nancy, Université de \\ Lorraine, CNRS UMR 7039, F-54506 Vandoeuvre-les-Nancy, France \\ (e-mail: didier.theilliol@univ-lorraine.fr)
}

\begin{abstract}
This paper deals with actuator fault estimation for a class of discrete-time nonlinear systems whose linear part is described by a Linear Parameter-Varying (LPV) form and nonlinear term is Lipschitz. In this paper, an augmented system is constructed by considering the fault as an auxiliary state vector. Then, a robust fault estimation observer is designed based on the augmented system. The proposed fault estimation observer is able to attenuate the effect of the fault variation and measurement noise and the observer design is formulated as a Linear Matrix Inequality (LMI) feasibility problem, which can be easily solved. Finally, a missile model is used to demonstrate the effectiveness of the proposed method.
\end{abstract}

Keywords: Actuator fault estimation, nonlinear systems, Linear Parameter-Varying (LPV), observer design, Linear Matrix Inequality (LMI).

\section{INTRODUCTION}

With the growing complexity of modern engineering systems and ever increasing demand for safety and reliability, fault diagnosis techniques have received great attention during the past decades. Generally, fault diagnosis approaches can be classified into three categories, i.e., modelbased methods, signal-based methods, and knowledgebased methods (Dai and Gao (2013)). Among these approaches, model-based fault diagnosis methods have been most intensively studied and various methods have been proposed in the literature, see e.g., Frank (1990), Chen and Patton (1999), Isermann (2005), Hwang et al. (2010), and the references therein.

As is known, most of the practical systems are nonlinear in nature. Therefore, there has been a lot of interest in fault diagnosis for nonlinear systems in the recent years. For example, Jiang et al. (2006) and Zhang et al. (2009) studied fault diagnosis for Lipschitz nonlinear systems by using adaptive observer. In Polycarpou and Helmicki (1995); Polycarpou and Trunov (2000), the online approximators are used to develop fault diagnosis methods for nonlinear systems. In addition, Proportional Integral (PI) observer and descriptor observer are also be used to develop fault diagnosis methods for nonlinear systems in the recent years, see e.g., Zhang et al. (2010), Astorga-Zaragoza et al. (2012), Gao and Ding (2007) etc. However, most of these results are dedicated to a specific

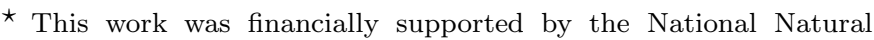
Science Foundation of China (Grant No. 61273162) and the Fundamental Research Funds for the Central Universities (Grant No. HIT.NSRIF.2015034).
}

class of nonlinear systems, i.e. Lipschitz nonlinear systems. In fact, fault diagnosis in nonlinear systems is still a difficult problem because there does not exist a universal methodology that can be applied to all nonlinear systems.

Since Linear parameter-varying (LPV) systems can be used to approximate nonlinear systems, the LPV representation provides a systematic and elegant way to deal with fault diagnosis for nonlinear systems. In recent years, a number of fault diagnosis methods have been proposed based on LPV models, e.g., Bokor and Balas (2004); Alwi et al. (2012); Rodrigues et al. (2013), etc. However, most of these methods are presented for continuous-time systems. In contrast to the continuous-time case, few results have been reported on fault diagnosis for discrete-time nonlinear systems. Nowadays, most of control systems are digitally implemented. Therefore, it is necessary to study the fault diagnosis design for discrete-time LPV systems.

On the other hand, when a nonlinear system with complex nonlinearity is considered, the corresponding LPV model will consists of a large number of linear models, which makes the fault diagnosis design complicated and also brings computation burden. Therefore, it is important to develop a more proper model to simplify the fault diagnosis design. In Boulkroune et al. (2013), the authors considered robust fault detection for a class of nonlinear descriptor systems whose nonlinear term is globally Lipschitz and linear term is described by a LPV form. However, the methods in Boulkroune et al. (2013) only address the fault detection problem but cannot be used to estimate the fault magnitudes. Here in our paper, the aim is not only to develop a fault detection method for discrete-time LPV 
systems with nonlinear terms but also to estimate actuator fault for such systems.

In this paper, a class of discrete-time nonlinear systems consists of a LPV term and Lipschitz nonlinearity are considered. The basic idea of this paper is to construct an augmented system by taking the fault as an auxiliary state vector. Then, a robust fault estimation observer is designed based on this augmented system. In this paper, the proposed fault estimation observer design is formulated as a set of Linear Matrix Inequalities (LMIs), which can be efficiently solved. The main contribution of this paper consists in the following aspects. First, a new fault estimation method is proposed for a class of discrete nonlinear systems which contains a LPV term and a Lipschitz nonlinear term. To the best of our knowledge, no work has been done on this subject. Moreover, fault variation and measurement noise are considered in the robust fault estimation observer design, which makes the proposed approach practical for real systems.

\section{PROBLEM FORMULATION}

Consider the following discrete-time nonlinear system

$$
\left\{\begin{array}{c}
x(k+1)=\sum_{i=1}^{h} \rho_{i}(\theta(k))\left[A_{i} x(k)+B_{i} u(k)+F_{i} f(k)\right] \\
+G \Phi(\theta(k), x(k)) \\
y(k)=C x(k)+H v(k)
\end{array}\right.
$$

where $x(k) \in \mathbb{R}^{n}$ is the state vector, $u(k) \in \mathbb{R}^{p}$ is the input vector, $f(k) \in \mathbb{R}^{q}$ is the fault vector, $y(k) \in \mathbb{R}^{m}$ denotes the measured output and $v(k) \in \mathbb{R}^{m}$ represents the measurement noise. $\Phi(\theta(k), x(k)) \in \mathbb{R}^{s}$ is a known nonlinear function. $A_{i} \in \mathbb{R}^{n \times n}, B_{i} \in \mathbb{R}^{n \times p}, F_{i} \in \mathbb{R}^{n \times q}$, $G \in \mathbb{R}^{n \times s}, C \in \mathbb{R}^{m \times n}$ and $H \in \mathbb{R}^{m \times n}$ are known constant matrices. $\theta(k) \in \mathbb{R}^{l}$ is a bounded time-varying parameter vector measurable online and not affected by fault, which is a general assumption in the LPV framework, see e.g. Boulkroune et al. (2013), Alwi et al. (2012) and Rodrigues et al. (2013). $\rho_{i}(\theta(k)), i=1,2, \ldots, h$ are the weighting functions which satisfy the following properties for all $k$ :

$$
\sum_{i=1}^{h} \rho_{i}(\theta(k))=1,0 \leq \rho_{i}(\theta(k)) \leq 1, i=1,2, \ldots, h
$$

Herein, $h$ is the total number of weighting functions.

Without loss of generality, it is assumed that $\operatorname{rank}(C)=$ $m, \operatorname{rank}\left(F_{i}\right)=q, q \leq m$. Moreover, the nonlinear function $\Phi(\theta(k), x(k))$ is assumed to satisfy the Lipschitz condition, i.e.

$\left\|\Phi\left(\theta(k), x_{1}(k)\right)-\Phi\left(\theta(k), x_{2}(k)\right)\right\| \leq L_{g}\left\|x_{1}(k)-x_{2}(k)\right\|(3)$

where $L_{g}>0$ denotes the Lipschitz constant.

The aim of this paper is to design an observer to obtain a fault estimation for $f(k)$.

To this end, the LPV system (1) is firstly formulated as an augmented system representation by letting $f(k)$ as a part of the augmented state vector, i.e.

$$
\bar{x}(k)=\left[\begin{array}{c}
x(k) \\
f(k)
\end{array}\right]
$$

Then, an augmented system is constructed as follows

$$
\left\{\begin{array}{c}
\bar{x}(k+1)=\sum_{i=1}^{h} \rho_{i}(\theta(k))\left[\bar{A}_{i} \bar{x}(k)+\bar{B}_{i} u(k)\right] \\
+\bar{D} \tilde{f}(k)+\bar{G} \Phi\left(\theta(k), C_{x} \bar{x}(k)\right) \\
y(k)=\bar{C} \bar{x}(k)+\operatorname{Hv}(k)
\end{array}\right.
$$

where

$$
\begin{gathered}
\bar{A}_{i}=\left[\begin{array}{cc}
A_{i} & F_{i} \\
0 & I_{q}
\end{array}\right], \bar{B}_{i}=\left[\begin{array}{c}
B_{i} \\
0
\end{array}\right], \bar{D}=\left[\begin{array}{c}
0 \\
I_{q}
\end{array}\right] \\
\bar{G}=\left[\begin{array}{c}
G \\
0
\end{array}\right], C_{x}=\left[\begin{array}{ll}
I_{n} & 0
\end{array}\right], \bar{C}=\left[\begin{array}{ll}
C & 0
\end{array}\right]
\end{gathered}
$$

and

$$
\tilde{f}(k)=f(k+1)-f(k)
$$

is the fault variation between two consecutive instants $k$ and $k+1$.

According to the definition of $\bar{x}(k)$ in (4), the actuator fault $f(k)$ can be estimated if the estimation of the augmented state $\bar{x}(k)$ is obtained. Based on this idea, this paper proposes the following fault estimation observer

$$
\begin{aligned}
\hat{\bar{x}}(k+1)= & \sum_{i=1}^{h} \rho_{i}(\theta(k))\left[\bar{A}_{i} \hat{\bar{x}}(k)+\bar{B}_{i} u(k)\right. \\
& \left.+L_{i}(y(k)-\bar{C} \hat{\bar{x}}(k))\right]+\bar{G} \Phi\left(\theta(k), C_{x} \hat{\bar{x}}(k)\right)
\end{aligned}
$$

where $\hat{\bar{x}}(k) \in \mathbb{R}^{(n+q)}$ is the estimate of the augmented state $\bar{x}(k) . L_{i} \in \mathbb{R}^{(n+q) \times m}, i=1,2, \ldots, h$ are matrices to be synthesized.

After the augmented state estimation $\hat{\bar{x}}(k)$ is obtained, the fault estimation can be determined as follows

$$
\hat{f}(k)=C_{f} \hat{\bar{x}}(k)
$$

where

$$
C_{f}=\left[\begin{array}{ll}
0 & I_{q}
\end{array}\right]
$$

Now, the problem is to design observer (9) such that the fault estimation $\hat{f}(k)$ is robust to the fault variation $\tilde{f}(k)$ and measurement noise $v(k)$. This is an $H_{\infty}$ design problem, which can be formulated as follows.

$\boldsymbol{H}_{\infty}$ fault estimation problem: Consider (5) and the fault estimation observer (9), the objective is to design that matrices $L_{i}, i=1,2, \ldots, h$ such that the fault estimation error $e_{f}(k)=f(k)-\hat{f}(k)$ robustly converges torwards zero, i.e.

$$
\left\|e_{f}\right\|_{2} \leq \sqrt{\gamma_{1}^{2}\|\tilde{f}\|_{2}^{2}+\gamma_{2}^{2}\|v\|_{2}^{2}+V(0)}
$$

where $\|\cdot\|_{2}$ represents the $\mathscr{L}_{2}$ norm, $\gamma_{1}$ and $\gamma_{2}$ are the disturbance attenuation levels: $\gamma_{1}$ is related to the robustness against the fault variation $\tilde{f}(k), \gamma_{2}$ represents the attenuation level of the measurement noise and $V(0)$ is a quadratic function which will be given latter.

\section{FAULT ESTIMATION OBSERVER DESIGN}

In this section, a solution to the $H_{\infty}$ fault estimation problem is proposed. 
First, a sufficient condition of the criterion (12) is given in the following Theorem.

Theorem 1. The criterion (12) is fulfilled if there exists a Lyapunov function $V(k)$ such that the following inequality holds for all $k$ :

$\Delta V(k)+e_{f}^{T}(k) e_{f}(k)-\gamma_{1}^{2} \tilde{f}^{T}(k) \tilde{f}(k)-\gamma_{2}^{2} v^{T}(k) v(k)<0$

where $\Delta V(k)=V(k+1)-V(k)$.

Proof. Define the following function

$$
J=V(\infty)-V(0)+\left\|e_{f}\right\|_{2}^{2}-\gamma_{1}^{2}\|\tilde{f}\|_{2}^{2}-\gamma_{2}^{2}\|v\|_{2}^{2}
$$

Note that $V(\infty) \geq 0$, it is obvious that the inequality $J<0$ implies

$$
\left\|e_{f}\right\|_{2}^{2}-\gamma_{1}^{2}\|\tilde{f}\|_{2}^{2}-\gamma_{2}^{2}\|v\|_{2}^{2}-V(0)<0
$$

i.e., the criterion (12) is satisfied.

Using the fact that $\Delta V(k)=V(k+1)-V(k)$, we obtain

$$
\begin{gathered}
J=\sum_{k=0}^{\infty} \Delta V(k)+\left\|e_{f}\right\|^{2}-\gamma_{1}^{2}\|\tilde{f}\|^{2}-\gamma_{2}^{2}\|v\|^{2} \\
=\sum_{k=0}^{\infty}\left[\Delta V(k)+e_{f}^{T}(k) e_{f}(k)-\gamma_{1}^{2} \tilde{f}^{T}(k) \tilde{f}(k)\right. \\
\left.\quad-\gamma_{2}^{2} v^{T}(k) v(k)\right]
\end{gathered}
$$

It can be seen that $J<0$ holds if (13) is fulfilled for all $k$. Then, it can be concluded that the criterion (12) is satisfied if (13) holds for all $k$.

Based on Theorem 1, the following Theorem is proposed to design the matrices $L_{i}, i=1,2, \ldots, h$ in observer (9). Theorem 2. For given scalars $\gamma_{1}>0$ and $\gamma_{2}>0$, if there exist a symmetric positive definite matrix $P \in$ $\mathbb{R}^{(n+q) \times(n+q)}$, a positive scalar $\eta>0$ and matrices $W_{i} \in$ $\mathbb{R}^{(n+q) \times m}, i=1,2, \ldots, h$ such that the following LMIs hold for all $i=1,2, \ldots, h$ :

$$
\left[\begin{array}{ccccc}
\mathscr{N}_{11} & 0 & 0 & 0 & \mathscr{N}_{15} \\
* & -\gamma_{1}^{2} I_{q} & 0 & 0 & \bar{D}^{T} P \\
* & * & -\gamma_{2}^{2} I_{m} & 0 & \mathscr{N}_{35} \\
* & * & * & -\eta I_{s} & \bar{G}^{T} P \\
* & * & * & * & -P
\end{array}\right]<0
$$

where

$$
\begin{gathered}
\mathscr{N}_{11}=-P+C_{f}^{T} C_{f}+\eta \bar{L}_{g}, \bar{L}_{g}=\left[\begin{array}{cc}
L_{g} I_{n} & 0 \\
0 & 0
\end{array}\right] \\
\mathscr{N}_{15}=\bar{A}_{i}^{T} P-\bar{C}^{T} W_{i}^{T}, \mathscr{N}_{35}=-H^{T} W_{i}^{T}
\end{gathered}
$$

Then the fault estimation observer (9) is robust against the fault variation and measurement noise, i.e. satisfying the criterion (12). Moreover, if the LMIs in (17) are solved, the matrices $L_{i}, i=1,2, \ldots, h$ are determined by

$$
L_{i}=P^{-1} W_{i}, i=1,2, \ldots, h
$$

Proof. Define the augmented state estimation error as

$$
e(k)=\bar{x}(k)-\hat{\bar{x}}(k)
$$

Using (1) and (9), the error dynamic is obtained as follows

$$
\begin{gathered}
e(k+1)=\sum_{i=1}^{h} \rho_{i}(\theta(k))\left[\left(\bar{A}_{i}-L_{i} \bar{C}\right) e(k)-L_{i} H v(k)\right] \\
+\bar{D} \tilde{f}(k)+\bar{G} \tilde{\Phi}(k)
\end{gathered}
$$

where $\tilde{\Phi}(k)$ is given by

$$
\tilde{\Phi}(k)=\Phi\left(\theta(k), C_{x} \bar{x}(k)\right)-\Phi\left(\theta(k), C_{x} \hat{\bar{x}}(k)\right)
$$

Take the following Lyapunov function

$$
V(k)=e^{T}(k) \operatorname{Pe}(k), P>0
$$

Substituting (22) into the difference of $V(k)$ gives

$$
\begin{aligned}
& \Delta V(k)=e^{T}(k) \mathscr{A}^{T}(k) P \mathscr{A}(k) e(k)-e^{T}(k) P e(k) \\
& \quad+2 e^{T}(k) \mathscr{A}^{T}(k) P[\bar{D} \tilde{f}(k)-\mathscr{L}(k) H v(k)+\bar{G} \tilde{\Phi}(k)] \\
& \quad+2 \tilde{f}^{T}(k) \bar{D}^{T} P[-\mathscr{L}(k) H v(k)+\bar{G} \tilde{\Phi}(k)] \\
& \quad+\tilde{f}^{T}(k) \bar{D}^{T} P \bar{D} \tilde{f}(k)-2 v^{T}(k) H^{T} \mathscr{L}^{T}(k) P \bar{G} \tilde{\Phi}(k) \\
& \quad+v^{T}(k) H^{T} \mathscr{L}^{T}(k) P \mathscr{L}(k) H v(k)+\tilde{\Phi}^{T}(k) \bar{G}^{T} P \bar{G} \tilde{\Phi}(k)
\end{aligned}
$$

where

$$
\begin{gathered}
\mathscr{A}(k)=\sum_{i=1}^{h} \rho_{i}(\theta(k))\left(T \bar{A}_{i}-L_{i} \bar{C}\right) \\
\mathscr{L}(k)=\sum_{i=1}^{h} \rho_{i}(\theta(k)) L_{i}
\end{gathered}
$$

Since $\Phi(\theta(k), x(k))$ satisfies the Lipschitz condition (3), it is easy to show that

$$
\eta \tilde{\Phi}^{T}(k) \tilde{\Phi}(k) \leq \eta e^{T}(k) \bar{L}_{g} e(k)
$$

where $\eta>0$ is a positive scalar and $\bar{L}_{g}$ is given by

$$
\bar{L}_{g}=\left[\begin{array}{cc}
L_{g} I_{n} & 0 \\
0 & 0
\end{array}\right]
$$

Using (25) and (28), we obtain

$$
\begin{aligned}
\Delta & V(k)+e_{f}^{T}(k) e_{f}(k)-\gamma_{1}^{2} \tilde{f}^{T}(k) \tilde{f}(k)-\gamma_{2}^{2} v^{T}(k) v(k) \\
\leq & e^{T}(k)\left[\mathscr{A}^{T}(k) P \mathscr{A}(k) e(k)-P+C_{f}^{T} C_{f}\right] e(k) \\
& +2 e^{T}(k) \mathscr{A}^{T}(k) P[\mathscr{G}(k) \tilde{f}(k)-\mathscr{L}(k) v(k)+\bar{H} \tilde{\Phi}(k)] \\
& +\tilde{f}^{T}(k)\left[\mathscr{G}^{T}(k) P \mathscr{G}(k)-\gamma_{1}^{2}\right] \tilde{f}(k) \\
& +2 \tilde{f}^{T}(k) \mathscr{G}^{T}(k) P[\bar{H} \tilde{\Phi}(k)-\mathscr{L}(k) v(k)] \\
& +v^{T}(k)\left[\mathscr{L}^{T}(k) P \mathscr{L}(k)-\gamma_{2}^{2} v^{T}(k) v(k)\right] v(k) \\
& -2 v^{T}(k) \mathscr{L}^{T}(k) P \bar{H} \tilde{\Phi}(k)+\tilde{\Phi}^{T}(k) \bar{H}^{T} P \bar{H} \tilde{\Phi}(k) \\
& +\eta e^{T}(k) \bar{L}_{g} e(k)-\eta \tilde{\Phi}^{T}(k) \tilde{\Phi}(k) \\
= & \xi^{T}(k) \Psi \xi(k)
\end{aligned}
$$

where

$$
\xi(k)=\left[\begin{array}{c}
e(k) \\
\tilde{f}(k) \\
v(k) \\
\tilde{\Phi}(k)
\end{array}\right], \Psi=\left[\begin{array}{cccc}
\Psi_{11} & \Psi_{12} & \Psi_{13} & \Psi_{14} \\
* & \Psi_{22} & \Psi_{23} & \Psi_{24} \\
* & * & \Psi_{33} & \Psi_{34} \\
* & * & * & \Psi_{44}
\end{array}\right]
$$

where

$$
\begin{gathered}
\Psi_{11}=\mathscr{A}^{T}(k) P \mathscr{A}(k)-P+C_{f}^{T} C_{f}+\eta \bar{L}_{g} \\
\Psi_{12}=\mathscr{A}^{T}(k) P \mathscr{G}(k), \Psi_{22}=\mathscr{G}^{T}(k) P \mathscr{G}(k)-\gamma_{1}^{2} I_{d} \\
\Psi_{13}=-\mathscr{A}^{T}(k) P \mathscr{L}(k), \Psi_{23}=-\mathscr{G}^{T}(k) P \mathscr{L}(k) \\
\Psi_{33}=\mathscr{L}^{T}(k) P \mathscr{L}(k)-\gamma_{2}^{2} I_{q}, \Psi_{14}=\mathscr{A}^{T}(k) P \bar{H} \\
\Psi_{24}=\mathscr{G}^{T}(k) P \bar{H}, \Psi_{34}=-\mathscr{L}^{T}(k) P \bar{H} \\
\Psi_{44}=\bar{H}^{T} P \bar{H}-\eta I_{s}
\end{gathered}
$$

From (30), it can be seen that (13) holds if $\Psi<0$. From Theorem 1, it is obtained that the criterion (12) is fulfilled if $\Psi<0$ is satisfied. 
By using Schur complement Lemma (Boyd et al. (1994)), $\Psi<0$ is equivalent to the following inequality

$$
\left[\begin{array}{ccccc}
\Psi_{11}^{\prime} & 0 & 0 & 0 & \mathscr{A}^{T}(k) P \\
* & -\gamma_{1}^{2} I_{q} & 0 & 0 & \bar{G}^{T} P \\
* & * & -\gamma_{2}^{2} I_{m} & 0 & -\mathscr{L}^{T}(k) P \\
* & * & * & -\eta I_{s} & \bar{H}^{T} P \\
* & * & * & * & -P
\end{array}\right]<0
$$

where $\Psi_{11}^{\prime}=-P+C_{f}^{T} C_{f}+\eta \bar{L}_{g}$.

Using the property of $\rho_{i}(\theta(k))$ in $(2)$, the above inequality (32) holds if the following matrix inequalities hold for all $i=1,2, \ldots, h$ :

$$
\left[\begin{array}{ccccc}
\mathscr{M}_{11} & 0 & 0 & 0 & \mathscr{M}_{15} \\
* & -\gamma_{1}^{2} I_{q} & 0 & 0 & \bar{G}^{T} P \\
* & * & -\gamma_{2}^{2} I_{m} & 0 & \mathscr{M}_{35} \\
* & * & * & -\eta I_{s} & \bar{H}^{T} P \\
* & * & * & * & -P
\end{array}\right]<0
$$

where

$$
\begin{gathered}
\mathscr{M}_{11}=-P+C_{f}^{T} C_{f}+\eta \bar{L}_{g} \\
\mathscr{M}_{15}=\left(\bar{A}_{i}-L_{i} \bar{C}\right)^{T} P, \mathscr{M}_{35}=-L_{i}^{T} P
\end{gathered}
$$

By letting $W_{i}=P L_{i}$, the inequalities in (33) become the LMIs in (17). Consequently, if the LMIs in (17) are solved, the matrices $L_{i}, i=1,2, \ldots, h$ are determined by (20).

Remark 1. In fact, the index $\gamma_{1}$ is related to the robustness against the fault variation $\tilde{f}(k)$ while $\gamma_{2}$ represents the attenuation level of the measurement noise. However, it should be noted that the robustness against fault variation and the insensitiveness to the measurement noise are conflicted. Therefore, it is necessary to take trade-off when choose $\gamma_{1}$ and $\gamma_{2}$ in fault estimation observer design.

\section{SIMULATIONS}

In this section, a simulation example is used to show the effectiveness of the proposed method. The system under consideration is a dynamic model of a missile from Lendek et al. (2010). The continuous-time model of the considered missile is given as follows

$$
\begin{gathered}
\dot{\alpha}=K_{\alpha} M C_{n}(\alpha, \delta, M) \cos (\alpha)+q \\
\dot{q}=K_{q} M^{2} C_{m}(\alpha, \delta, M)
\end{gathered}
$$

where $M$ denotes the Mach number, which is an exogenous scheduling variable, $\alpha$ is the angle of attack, $q$ is the pitch rate and $\delta$ is the action of the tail fin actuator. It is assumed that $\alpha$ and $q$ can be measured. The coefficients in (34)-(35) are expressed as

$$
\begin{gathered}
K_{\alpha}=(\pi / 180) 0.7 P_{0} S /\left(m v_{s}\right), K_{q}=(\pi / 180) 0.7 P_{0} S d / I_{y} \\
C_{n}(\alpha, \delta, M)=a_{n} \alpha^{3}+b_{n}|\alpha| \alpha+c_{n}(2-M / 3) \alpha+d_{n} \delta \\
C_{m}(\alpha, \delta, M)=a_{m} \alpha^{3}+b_{m}|\alpha| \alpha+c_{n}(-7+8 M / 3) \alpha+d_{m} \delta
\end{gathered}
$$

where $P_{0}=973.3 \mathrm{lbs} / \mathrm{ft}^{2}$ is the static pressure at $20000 \mathrm{ft}$, $S=0.44 \mathrm{ft}^{2}$ is the surface area, $v_{s}=1036.4 \mathrm{ft} / \mathrm{s}$ is the speed of sound at $20000 \mathrm{ft}, d=0.75 \mathrm{ft}$ is the diameter, $I_{y}=182.5 \mathrm{slug} \cdot \mathrm{ft}^{2}$ is the pitch moment of inertia and $m=$
13.98 slugs is the mass of the missile. Other parameters in $C_{n}(\alpha, \delta, M)$ and $C_{m}(\alpha, \delta, M)$ are given as follows.

$$
\begin{gathered}
a_{n}=0.000103 \mathrm{deg}^{-3}, b_{n}=-0.00945 \mathrm{deg}^{-2} \\
c_{n}=0.1696 \mathrm{deg}^{-1}, d_{n}=-0.034 \mathrm{deg}^{-1} \\
a_{m}=0.000215 \mathrm{deg}^{-3}, b_{m}=-0.0195 \mathrm{deg}^{-2} \\
c_{m}=0.051 \mathrm{deg}^{-1}, d_{m}=-0.206 \mathrm{deg}^{-1}
\end{gathered}
$$

Denote $x=\left[\begin{array}{ll}\alpha & q\end{array}\right]^{T}, u=\delta$, the dynamic equations (34)(35) are written as

$$
\dot{x}=\mathcal{A}_{c}(M, \alpha) x+\mathcal{B}_{c}(M, \alpha) u+\Phi_{c}(M, \alpha)
$$

where

$$
\begin{aligned}
& \mathcal{A}_{c}(M, \alpha)=\left[\begin{array}{cc}
K_{\alpha} M \cos (\alpha) c_{n}(2-M / 3) & 1 \\
K_{q} M^{2} c_{m}(-7+8 M / 3) & 0
\end{array}\right] \\
& \mathcal{B}_{c}(M, \alpha)=\left[\begin{array}{c}
K_{\alpha} M \cos (\alpha) d_{n} \\
K_{q} M^{2} d_{m}
\end{array}\right] \\
& \Phi_{c}(M, \alpha)=\left[\begin{array}{c}
K_{\alpha} M\left(a_{n} \alpha^{3}+b_{n}|\alpha| \alpha\right) \cos (\alpha) \\
K_{q} M^{3}\left(a_{m} \alpha^{3}+b_{m}|\alpha| \alpha\right)
\end{array}\right]
\end{aligned}
$$

Notice that $K_{\alpha}$ is very small, then the above system (36) can be approximated by

$$
x(k+1)=\tilde{\mathcal{A}}_{c}(M) x(k)+\tilde{\mathcal{B}}_{c}(M) u(k)+\Phi_{c}(M, \alpha)
$$

where

$$
\begin{gathered}
\tilde{\mathcal{A}}_{c}(M)=\left[\begin{array}{cc}
0 & 1 \\
K_{q} M^{2} c_{m}(-7+8 M / 3) & 0
\end{array}\right] \\
\tilde{\mathcal{B}}_{c}(M)=\left[\begin{array}{c}
0 \\
K_{q} M^{2} d_{m}
\end{array}\right]
\end{gathered}
$$

By using the Euler approximation method (Arcak and Nešić (2003); Mao et al. (2010)), the continuous system (37) is discretized as

$$
x(k+1)=\mathcal{A}(M) x(k)+\mathcal{B}(M) u(k)+\Phi(M, \alpha)
$$

where

$$
\begin{gathered}
\mathcal{A}(M)=t_{s} \tilde{\mathcal{A}}_{c}(M)+I_{3} \\
\mathcal{B}(M)=t_{s} \tilde{\mathcal{B}}_{c}(M) \\
\Phi(M, \alpha)=t_{s} \Phi_{c}(M, \alpha)
\end{gathered}
$$

and $t_{s}=0.2$ is the sampling period. In this paper, the range of $M$ is given by

$$
M \in[2,4]
$$

Moreover, it is assumed $\alpha$ is bounded as $\alpha \in[-15,15]$. Then, it can be derived that the nonlinear function $\Phi(M, \alpha)$ satisfies the Lipschitz condition (3) with $L_{g}=$ 0.04 .

In order to use the proposed method, the discretized system (38) should be written as an LPV representation with a nonlinear term. Using the methodology in Hamdi et al. (2012), the following polytopic system is constructed to approximate system (38):

$$
\begin{gathered}
x(k+1)=\sum_{i=1}^{2} \rho_{i}(\theta(k))\left[A_{i} x(k)+B_{i} u(k)+F_{i} f(k)\right] \\
+G \Phi(\theta(k), x(k))
\end{gathered}
$$


where the parameter vector is $\theta(k)=M$ and the weighting functions $\rho_{i}(\theta(k)), i=1,2$ are described as follows

$$
\begin{aligned}
& \rho_{1}(\theta(k))=\frac{\bar{M}-M}{\bar{M}-\underline{M}} \\
& \rho_{2}(\theta(k))=\frac{M-\underline{M}}{\bar{M}-\underline{M}}
\end{aligned}
$$

Herein, $\underline{M}$ and $\bar{M}$ are the extreme values of the parameter $M$, i.e.

$$
\underline{M}=2, \bar{M}=4
$$

The matrices in system (39) are given by

$$
\begin{gathered}
A_{1}=\left[\begin{array}{ll}
1.0000 & 0.2000 \\
0.0033 & 1.0000
\end{array}\right], A_{2}=\left[\begin{array}{cc}
1.0000 & 0.2000 \\
-0.0292 & 1.0000
\end{array}\right] \\
B_{1}=\left[\begin{array}{c}
0.0000 \\
-0.0081
\end{array}\right], B_{2}=\left[\begin{array}{c}
0.0000 \\
-0.0322
\end{array}\right] \\
G=\left[\begin{array}{ll}
1 & 0 \\
0 & 1
\end{array}\right], F_{i}=B_{i}, i=1,2
\end{gathered}
$$

In addition, it is assumed that the measurement equation is described by

$$
y(k)=C x(k)+H v(k)
$$

where

$$
C=\left[\begin{array}{ll}
1 & 0 \\
0 & 1
\end{array}\right], H=\left[\begin{array}{cc}
0.01 & 0 \\
0 & 0.01
\end{array}\right]
$$

Now, a nonlinear system in the form of (1) is obtained by combining (39) and (40). Then, the proposed method can be applied to design a fault estimation observer.

Remark 2. Note that the fault distribution matrices $F_{i}$, which are the same as $B_{i}$, are very small. From the perspective of inverse problem, the fault estimation problem is not well-posed. This implies that the fault estimation will be severely effected by the measurement noise. Therefore, it is necessary to consider the influence of measurement noise and design a robust fault estimation method.

By choosing $\gamma_{1}=7, \gamma_{2}=1.4$ and solving the LMIs in (17), we obtain the following matrices in the fault estimation observer (9)

$$
L_{1}=\left[\begin{array}{cc}
0.6195 & 0.2218 \\
0.0253 & 1.2218 \\
0.0295 & -28.1344
\end{array}\right], L_{2}=\left[\begin{array}{cc}
0.6194 & 0.2219 \\
0.0025 & 1.9049 \\
0.0507 & -28.2126
\end{array}\right]
$$

In the simulation, the initial condition of the nonlinear system is $x(0)=\left[\begin{array}{ll}10 & 2\end{array}\right]^{T}$. The time-varying variable $M$ is shown in Fig. 1 and the weighting functions are depicted in Fig . 2. In addition, the measurement noise used in the simulation is zero-mean Gaussian sequences with standard deviation $\sigma=0.01$.

First, consider an abrupt fault which is represented by

$$
f(k)=\left\{\begin{array}{cc}
0 & k<150 \\
15 & k \geq 150
\end{array}\right.
$$

The fault estimation result of the robust fault estimation observer is depicted in Fig. 3. Therein, the actual fault is depicted by solid line and the fault estimation is represented by the dash one. As shown in Fig. 3, the proposed

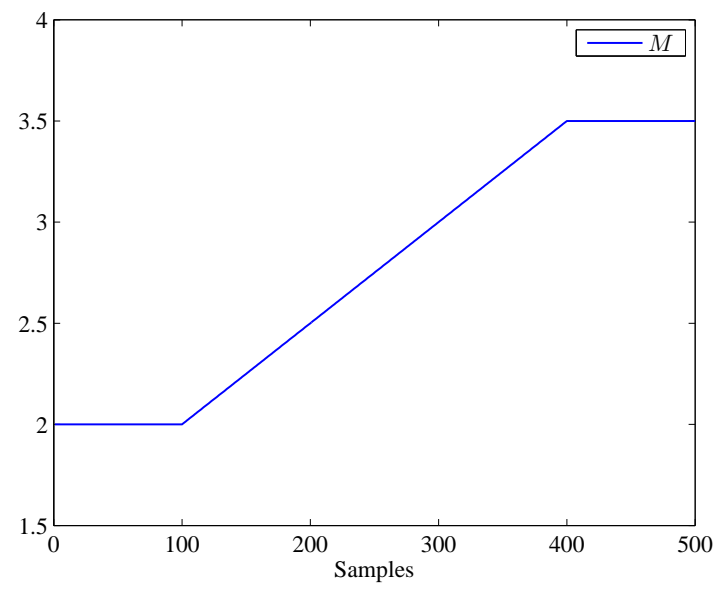

Fig. 1. The Mach number $M$ in the simulation

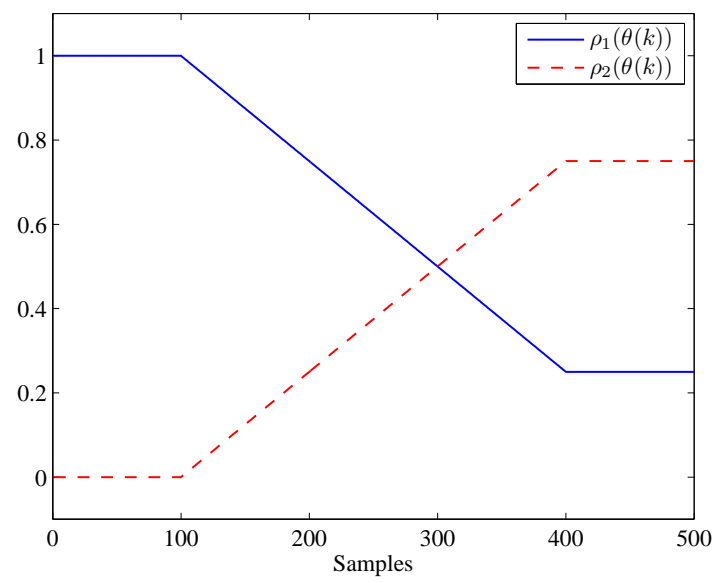

Fig. 2. The weighting functions $\rho_{1}(\theta(k))$ and $\rho_{2}(\theta(k))$

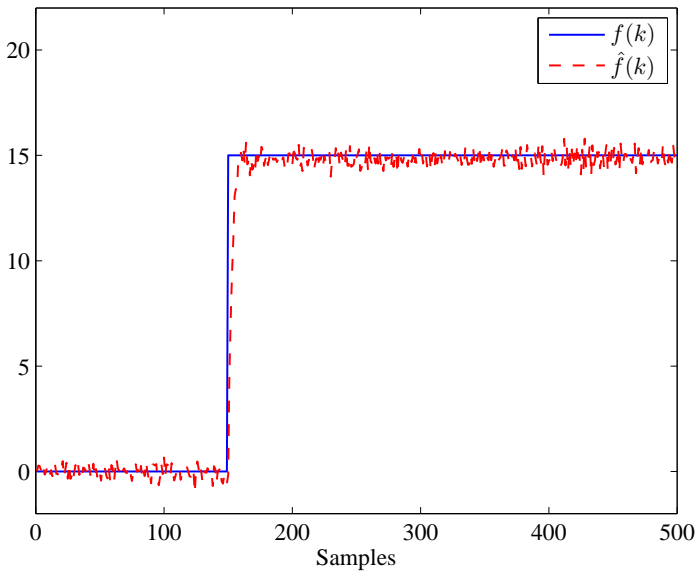

Fig. 3. The fault estimation result in an abrupt fault situation 
fault estimation observer provides accurate fault estimation in the presence of measurement noise. To illustrate the performance of robust fault estimation observer in dealing with time-varying fault, the following fault is also simulated

$$
f(k)=\left\{\begin{array}{cc}
0 & k<100 \\
10 \sin (0.05(k-100)) & k \geq 100
\end{array}\right.
$$

In this situation, the fault estimation result is depicted in Fig. 4. As shown in Fig. 4, the proposed fault estimation observer also exhibits satisfactory performance in the timevarying fault scenario.

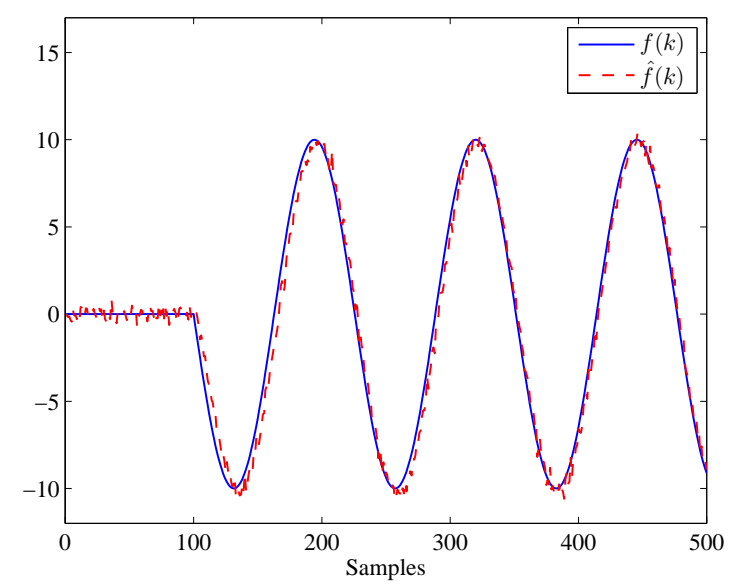

Fig. 4. The fault estimation result in a time-varying fault situation

\section{CONCLUSION}

A new actuator fault diagnosis technique capable of estimating actuator faults for a class of nonlinear systems has been proposed in this paper. The considered nonlinear system consists of an LPV part and a Lipschtiz nonlinear term. By considering the fault as an auxiliary state vector, an augmented system is first constructed. Based on the augmented system, a robust fault estimation observer is designed to attenuate the effect of the fault variation and measurement noise. The effectiveness of the proposed method is demonstrated by a missile model.

\section{REFERENCES}

X. Dai, Z. Gao. From model, signal to knowledge: A data-driven perspective of fault detection and diagnosis. IEEE Transcations on Industrial Informatics, 6(4): 2226-2238, 2013.

P. M. Frank. Fault diagnosis in dynamic systems using analytical and knowledge based redundancy-A survey and some new results. Automatica, 26(3):459-474, 1990.

J. Chen and R. Patton. Robust Model-Based Fault Diagnosis for Dynamic Systems. Kluwer Academic Publishers, Boston, 1999.

R. Isermann. Model-Based Fault-Detection and DiagnosisStatus and Applications. Annual Reviews in Control, 29(1):71-85, 2005.

I. Hwang, S. Kim, Y. Kim, C. E. Seah. A survey of fault detection, isolation, and reconfiguration methods. IEEE
Transactions on Control Systems Technology, 18(3): 636-653, 2010.

B. Jiang, M. Staroswiecki, V. Cocquempot. Fault accommodation for nonlinear dynamic systems. IEEE Transactions on Automatic Control, 51(9):1578-1583, 2006.

K. Zhang, B. Jiang, P. Shi. Fast fault estimation and accommodation for dynamical systems. IET Control Theory \& Applications, 3(2):189-199, 2009.

M. M. Polycarpou and A. J. Helmicki. Automated fault detection and accommodation: A learning systems approach. IEEE Transactions on Systems, Man and Cybernetics, 25(11):1447-1458, 1995.

M. M. Polycarpou and A. B. Trunov. Learning approach to nonlinear fault diagnosis: Detectability analysis. IEEE Transactions on Automatic Control, 45(4): 806-812, 2000.

K. Zhang, B. Jiang, P. Shi. Observer-based integrated robust fault estimation and accommodation design for discrete-time systemss. International Journal of Control, 83(6):1167-1181, 2010.

C. M. Astorga-Zaragoza,D. Theilliol, J. C. Ponsart, M. Rodrigues. Fault diagnosis for a class of descriptor linear parameter-varying systems. International Journal of Adaptive Control and Signal Processing, 26(3):208-223, 2012.

Z. Gao and S. X. Ding. Sensor fault reconstruction and sensor compensation for a class of nonlinear state-space systems via a descriptor system approach. IET Control Theory \& Applications, 1(3):578-585, 2007.

J. Bokor and G. Balas. Detection filter design for LPV systems-A geometric approach. Automatica, 40(3):511$518,2004$.

H. Alwi, C. Edwards, A. Marcos. Fault reconstruction using a LPV sliding mode observer for a class of LPV systems. Journal of the Franklin Institute, 349(2):510530, 2012.

M. Rodrigues, M. Sahnoun, D. Theilliol, J. C. Ponsart. Sensor fault detection and isolation filter for polytopic LPV systems: A winding machine application. Journal of Process Control, 23(6):805-816, 2013.

B. Boulkroune, S. Haliabi, A. Zemouche. $H_{-} / H_{\infty}$ fault detection filter for a class of nonlinear descriptor systems. International Journal of Control, 86(2):253-262, 2013.

Zs. Lendeka, J. Lauber, T. M. Guerra, R. Babuka, B. DeSchutter. Adaptive observers for TS fuzzy systems with unknown polynomial inputs. Fuzzy Sets and Systems, 161(15):2043-2065, 2010.

M. Arcak and D. Nešić. Observer design for sampleddata nonlinear systems via approximate discrete-time models. Proceedings of the 42nd IEEE Conference on Decision and Control, pages 49-54, December 2004.

Z. Mao, B. Jiang, P. Shi. Fault-tolerant control for a class of nonlinear sampled-data systems via a Euler approximate observer. Automatica, 46(11):1852-1859, 2010.

H. Hamdi, M. Rodrigues, C. Mechmeche, D. Theilliol, N. B. Braiek. Fault detection and isolation in linear parameter-varying descriptor systems via proportional integral observer. International Journal of Adaptive Control and Signal Processing, 26(3):224-240, 2012. 